\title{
重复取样条件下的点格局分析
}

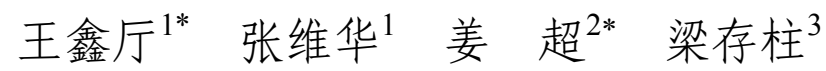 \\ ${ }^{1}$ 内蒙古工业大学能源与动力工程学院, 呼和浩特 $010051 ;{ }^{2}$ 中国农业科学院草原研究所/农业部草地生态与修复治理重点实验室, 呼和浩特 010010 ; \\ ${ }^{3}$ 内蒙古大学生态与环境学院, 呼和浩特 010021
}

摘 要 种群空间格局是生态学研究的基本内容, 点格局是研究种群格局最常用的方法。重复取样是生态学研究过程中所遵 循的基本原则。但是, 在应用点格局分析种群格局的研究实例中, 重复取样的案例非常少见。鉴于重复取样对于点格局研究 的重要性, 该文引入了重复取样条件下点格局的统计学分析方法, 并计算分析了典型草原带过度放牧引起的严重退化群落在 恢复演替8年阶段羊草(Leymus chinensis)与大针茅(Stipa grandis)两个优势种群在单一取样与重复取样条件下的格局特征。研 究结果表明, 各个重复之间格局特征存在差异, 这种差异说明由单一取样得到的种群格局特征属于特例而不具有代表性, 因 此得到的结论值得推敲。整合多个重复取样条件下的点格局是多个重复条件下的种群格局的平均状态, 这种状态更能体现格 局的整体特征而具代表性。

关键词 重复取样; 点格局分析; O-Ring函数

引用格式: 王金原, 张维华, 姜超, 梁存柱 (2017). 重复取样条件下的点格局分析. 植物生态学报, 41, 577-584. doi: 10.17521/cjpe.2016.0383

\section{Point pattern analysis under conditions of replicated sampling}

\author{
WANG Xin-Ting ${ }^{1 *}$, ZHANG Wei-Hua ${ }^{1}$, JIANG Chao ${ }^{2 *}$, and LIANG Cun-Zhu ${ }^{3}$ \\ ${ }^{1}$ School of Energy and Power Engineering, Inner Mongolia University of Technology, Huhhot 010051, China; ${ }^{2}$ Institute of Grassland Research, Chinese \\ Academy of Agriculture Sciences, Key of Laboratory of Grassland Ecology and Restoration, Ministry of Agriculture, Huhhot 010010, China; and ${ }^{3}$ College of \\ Ecology and Environment, Inner Mongolia University, Huhhot 010021, China
}

\begin{abstract}
Aims The analysis of point patterns, which deals with data sets consisting of mapped locations of organisms in a study region, is especially important to plant ecological studies because the locations of plants can often be approximated as points. However, few studies used point pattern analysis with data collected by replicated sampling - a principle procedure of acquiring data in ecological research. Therefore, we explore the applicability of point pattern analysis under conditions of replicated sampling in this study.

Methods Three replicated $5 \mathrm{~m} \times 5 \mathrm{~m}$ plots of homogenous communities were established on a site with eight years of restoration in Nei Mongol steppe. In each plot, the locations of individuals in Leymus chinensis and Stipa grandis populations were mapped. O-Ring function was used to describe the population patterns and species association between L. chinensis and S. grandis for each plot as well as the integrative data of the three replicates.

Important findings Population patterns and species associations differed among the three replicated plots. This illustrates that if point pattern analysis was applied to describe the population patterns and species associations only by using data from a single plot sampling, the results could be misleading. Whereas it would be more reliable to integrate the data of replicated plots in the point pattern analysis because in this way the resulting O-Ring function is a weighted average, where the weight is the number of points in the replicate $i$ divided by the total number of points in all replicated plots.
\end{abstract}

Key words replicated sampling; point pattern analysis; O-Ring function

Citation: Wang XT, Zhang WH, Jiang C, Liang CZ (2017). Point pattern analysis under conditions of replicated sampling. Chinese Journal of Plant Ecology, 41, 577-584. doi: 10.17521/cjpe.2016.0383

重复取样是生态学家研究生态学问题时在实 验设计过程中所遵循的基本原则。种群空间格局与 过程是生态学研究的一个基本问题，一直以来备受
关注(Watt, 1947; Greig-Smith, 1952, 1987; Kershaw, 1963; Pielou, 1968; Galiano, 1983; Dale \& MacIsaac, 1989; ver Hoef et al., 1993; Condit et al., 2000;

收稿日期Received: 2016-12-19 接受日期Accepted: 2017-04-05

* 共同通信作者 Co-author for correspondence (E-mail: wang_x_t2002@163.com; jcfly2004@126.com) 
Wiegand et al., 2007; Wang et al., 2010, 2014; Jácome-Flores et al., 2016), 成为生态学研究中长盛 不衰的课题。在研究种群空间格局与过程时, 点格 局成为最重要、最常用的方法(Wiegand \& Moloney, 2004; Velázquez et al., 2016)。可是，在应用点格局 探究种群空间格局的过程中, 重复取样却非常鲜见 (e.g., Graff \& Aguiar, 2011)。由于没用重复取样, 实 验结果可能是特例而不具有普适意义, 这样得到的 结论其可靠程度就值得推敲, 因此, 一些学者在文 章投稿时可能会因为实验设计没有重复而遭拒稿。 尽管关于重复取样条件下的点格局分析的统计学方 法(Diggle，2003)和计算程序(Programita 2010版, www.thorsten-wiegand.de/towi_programita.html/) 已 有论述, 可是并未引起生态学研究者的重视, 这一 方面可能是习惯所致, 一直以来在应用点格局研究 种群格局时基本上都是单一取样; 另一方面，也可 能是重复取样工作量巨大, 致使研究者不想设计重 复取样。无论如何, 在重复取样条件下, 应用点格局 分析探讨种群格局具有重要的生态学意义。本文提 出点格局研究中重复取样的问题, 并通过研究实 例论述之, 期望引起广大生态学同仁的重视并加以 推广。

\section{1 重复取样条件下点格局的计算}

点格局是植物种群空间格局研究中广泛使用 的方法, 其基础数据为种群个体在研究区域内的平 面坐标。Ripley's $K$ 函数 $(K(r))$ 为点格局分析的基本 方法, 在此基础上发展了成对相关函数 $(g(r))$ 和 O-Ring函数 $(O(r))$, 三者之间的关系如下: $g(r)=$ $(2 \pi r)^{-1} d K(r) / d r, O(r)=\lambda g(r)$ (Wiegand \& Moloney, 2004)。由于 $K(r)$ 与 $g(r)$ 在较多文献中均有描述而 $O(r)$ 相对较少, 本文选择 $O(r)$ 加以介绍。(对于 $K(r)$ 函数, $N$ 个重复取样条件下的数学表达式见附录)

O-Ring函数的基本数学表达式:

$$
O^{w}(r)=\frac{\frac{1}{n} \sum_{i=1}^{n} \operatorname{Points}\left[R_{i}^{w}(r)\right]}{\frac{1}{n} \sum_{i=1}^{n} \operatorname{Area}\left[R_{i}^{w}(r)\right]}
$$

式中, $n$ 为研究区域内点(植物个体)的数量; $R_{i}^{w}(r)$ 为以 第 $i$ 点为圆心 $r$ 为半径、 $w$ 为带宽的圆环; $\operatorname{Points}\left[R_{i}^{w}(r)\right]$ 表示圆环内点的数量; Area $\left[R_{i}^{w}(r)\right]$ 表
示圆环的面积。

应用式(1)可以直接计算单一取样条件下的种 群格局。对于 $N$ 个重复取样，可以在式(1)基础上扩 展, 得到如下数学表达式:

$$
O^{w}(r)=\frac{\left(\begin{array}{c}
\frac{n^{1}}{M}\left(\frac{1}{n^{1}} \sum_{i^{1}=1}^{n^{1}} \operatorname{Points}\left[R_{i^{1}}^{w}(r)\right]\right)+\cdots+ \\
\frac{n^{j}}{M}\left(\frac{1}{n^{j}} \sum_{i^{j}=1}^{n^{j}} \operatorname{Points}\left[R_{i^{j}}^{w}(r)\right]\right)+\cdots+ \\
\frac{n^{N}}{M}\left(\frac{1}{n^{N}} \sum_{i^{N}=1}^{n^{N}} \operatorname{Points}\left[R_{i^{N}}^{w}(r)\right]\right)
\end{array}\right)}{\left(\begin{array}{c}
\frac{n^{1}}{M}\left(\frac{1}{n^{1}} \sum_{i^{1}=1}^{n^{1}} \operatorname{Area}\left[R_{i^{1}}^{w}(r)\right]\right)+\cdots+ \\
\frac{n^{j}}{M}\left(\frac{1}{n^{j}} \sum_{i^{j}=1}^{n^{j}} \operatorname{Area}\left[R_{i^{j}}^{w}(r)\right]\right)+\cdots+ \\
\frac{n^{N}}{M}\left(\frac{1}{n^{N}} \sum_{i^{N}=1}^{n^{N}} \operatorname{Area}\left[R_{i^{N}}^{w}(r)\right]\right)
\end{array}\right)}
$$

式中, $i^{j}$ 为种群在第 $j$ 个重复中的第 $i$ 个点; $n^{j}$ 为种群在 第 $j$ 个重复中的个体数; $M$ 为种群在所有重复中的个 体总数, $M=\Sigma_{j} n^{j}, N$ 指重复取样数。

式(2)可化简为:

$$
\begin{gathered}
\sum_{i^{1}=1}^{n^{1}} \operatorname{Points}\left[R_{i^{1}}^{w}(r)\right]+\cdots+ \\
\sum_{i^{j}=1}^{n^{j}} \operatorname{Points}\left[R_{i^{j}}^{w}(r)\right]+\cdots+ \\
O^{w}(r)=\frac{\sum_{i^{N}=1}^{n^{N}} \operatorname{Points}\left[R_{i^{N}}^{w}(r)\right]}{\sum_{i^{1}=1}^{n^{1}} \operatorname{Area}\left[R_{i^{1}}^{w}(r)\right]+\cdots+} \\
\sum_{i^{j}=1}^{n^{j}} \operatorname{Area}\left[R_{i^{j}}^{w}(r)\right]+\cdots+ \\
\sum_{i^{N}=1}^{n^{N}} \operatorname{Area}\left[R_{i^{N}}^{w}(r)\right]
\end{gathered}
$$

由于通过 $O(r)$ 既可以分析单一种群的格局特征, 又可以分析两个物种的种间关联, 为了区分两种过 程，通常用 $O_{11}(r)$ 表示单一种群，而用 $O_{12}(r)$ 表示两 个物种, $O_{11}(r)$ 与 $O_{12}(r)$ 的 $N$ 个重复结果的数学表达 式分别为: 


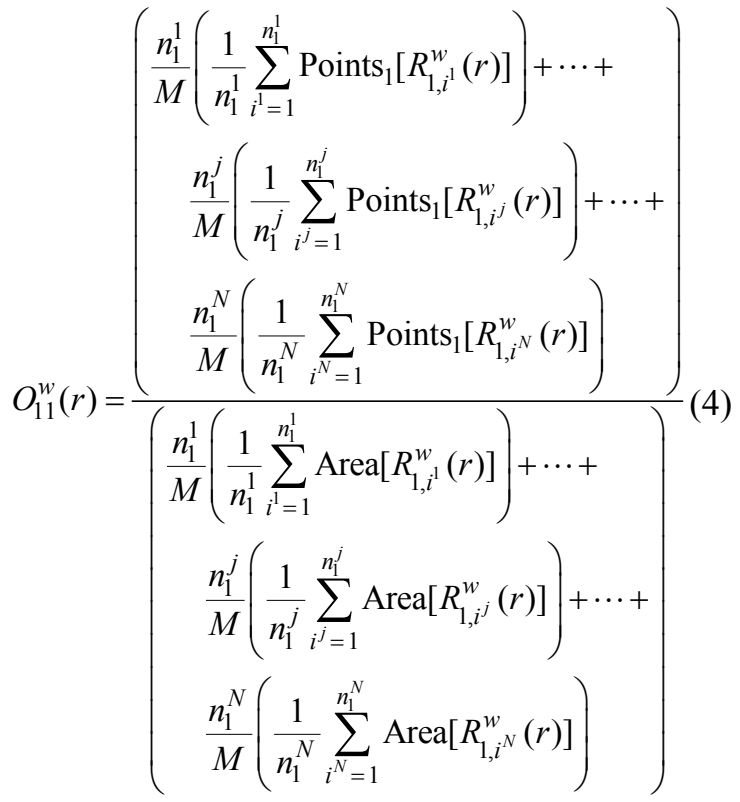

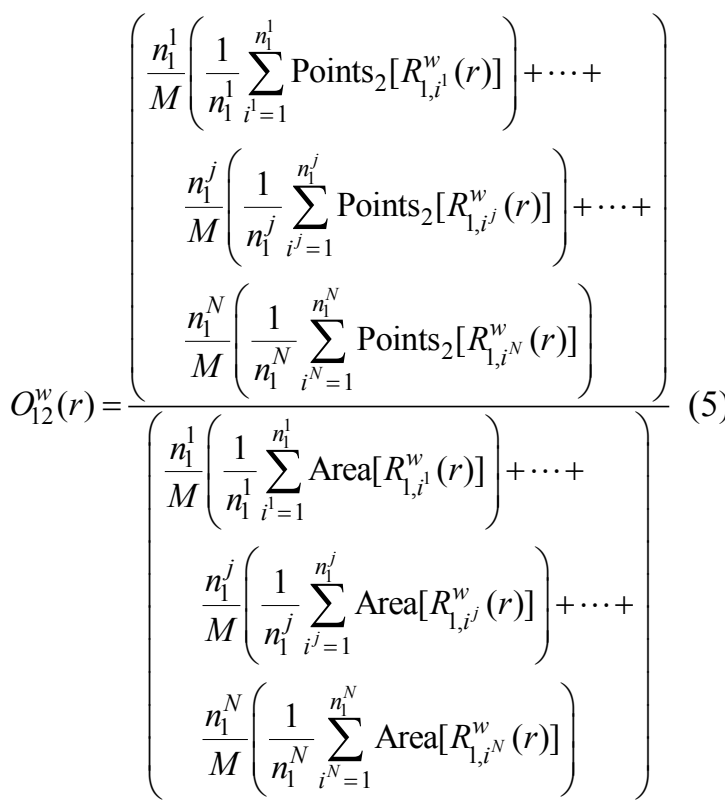

可化简为:

$$
\begin{gathered}
\sum_{i^{1}=1}^{n_{1}^{1}} \operatorname{Points}_{1}\left[R_{1, i^{1}}^{w}(r)\right]+\cdots+ \\
\sum_{11}^{w}(r)=\frac{\sum_{i^{j}=1}^{n_{1}^{j}} \operatorname{Points}_{1}\left[R_{1, i^{j}}^{w}(r)\right]+\cdots+}{\sum_{i^{1}=1}^{n_{1}^{1}} \operatorname{Points}_{1}\left[R_{1, i^{N}}^{w}(r)\right]} \\
\sum_{i^{j}=1}^{n_{1}^{N}} \operatorname{Area}\left[R_{1, i^{1}}^{w}(r)\right]+\cdots+ \\
\sum_{i^{N}=1}^{n_{1}^{N}} \operatorname{Area}\left[R_{1, i^{j}}^{w}(r)\right]+\cdots+
\end{gathered}
$$

$$
\begin{gathered}
\sum_{i^{1}=1}^{n_{1}^{1}} \operatorname{Points}_{2}\left[R_{1, i^{1}}^{w}(r)\right]+\cdots+ \\
\sum_{i^{j}=1}^{n_{1}^{j}} \operatorname{Points}_{2}\left[R_{1, i^{j}}^{w}(r)\right]+\cdots+ \\
\sum_{i^{1}=1}^{n_{1}^{1}=1} \operatorname{Area}_{1}^{n_{1}^{N}} \operatorname{Points}_{2}\left[R_{1, i^{\prime}}^{w}(r)\right]+\cdots+ \\
\sum_{i^{j}=1}^{n_{1}^{j}} \operatorname{Area}^{{ }^{N}}\left[R_{1, i^{j}}^{w}(r)\right]+\cdots+ \\
\sum_{i^{N}=1}^{n_{1}^{N}} \operatorname{Area}\left[R_{1, i^{N}}^{w}(r)\right]
\end{gathered}
$$

其中, 下角标 1 是指种群 1 ; 下角标 2 是指种群 2 。在 式(4)与式(6)中, Points 1 [X]表示在区域X (以物种1第 $i$ 个体为圆心、 $r$ 为半径、 $w$ 为带宽的圆环)内物种 1 的 个体数; 在式(5)与式(7)中, Points $2[X]$ 表示在区域 $\mathrm{X}$ (以物种 1 第 $i$ 个体为圆心、 $r$ 为半径、 $w$ 为带宽的圆环) 内物种 2 的个体数。

应用 O-Ring 函数分析种群空间格局与应用 Ripley's $K$ 函数类似, 通常将实测数据的 $O(r)$ 结果与 零模型(null models)模拟得到的置信区间进行对比, 从而判断格局特征。一般来说, 零模型通常选择完 全空间随机模型(CSR)。这样, 就单一种群 $O_{11}(r)$ 来 说, 实测结果如果位于置信区间之内, 就意味着种 群为随机分布; 若向上偏离置信区间上限, 说明种 群为聚集分布; 而向下偏离置信区间下限，则表明 种群为均匀分布。对种间关联 $O_{12}(r)$ 而言, 实测结果 若位于置信区间内，则表明两个物种之间没有关系; 倘若偏离置信区间, 就会出现两种情况: 位于上置信 区间之上——正关联; 位于下置信区间之下一一负 关联。

\section{2 研究实例}

\section{1 试验设计}

研究实例来源于内蒙古典型草原区，试验于 2004年7月在内蒙古锡林郭勒盟典型草原地带中国 科学院草原生态系统定位研究站设置的围栏样地内 进行。样地位于丘陵坡麓前缘与锡林河二级阶地之 间，地势微倾斜，地表较平整，土壤为典型栗钙土， 地理坐标为 $43.63^{\circ} \mathrm{N}, 116.70^{\circ} \mathrm{E}$, 平均海拔 $1187 \mathrm{~m}$ 。 
该地区属温带大陆性半干旱气候, 冬季寒冷干燥, 夏季温暖较湿润, 年平均气温 $0.18{ }^{\circ} \mathrm{C}$; 年降水量 $349.6 \mathrm{~mm}$, 年平均净水面蒸发量 $1641.5 \mathrm{~mm}$, 年日 照时数 $2533 \mathrm{~h}$, 大于 $0{ }^{\circ} \mathrm{C}$ 的积温为 $2428.7{ }^{\circ} \mathrm{C}$, 大于 $10{ }^{\circ} \mathrm{C}$ 的多年平均积温为 $1983.3{ }^{\circ} \mathrm{C}$, 具有冬寒夏温 的中温带气候特征(王金金厅等, 2013)。本项研究选择 1996年围封的退化群落恢复样地, 该样地围封时处 于严重退化状态的冷蒿+粘隐子草(Artemisia frigida + Cleistogenes squarrosa) 群落阶段, 属于羊草+大针 茅(Leymus chinensis + Stipa grandis $)$ 草原的严重退化 变体, 面积 $50 \mathrm{~m} \times 400 \mathrm{~m}$ (王金金厅等, 2013)。至测定时, 样地已恢复 8 年。

本试验采用摄影定位法(王金金厅等，2006)记录 了上述样地中羊草和大针茅 2 个优势物种的空间数 据, 为了消除生境异质性对实验的影响, 在空间数 据测定时，选定地表平坦、群落外貌均匀且具有代 表性的 3 个 $5 \mathrm{~m} \times 5 \mathrm{~m}$ 的群落片段, 即重复取样 3 次, 其种群个体数量和位点分别见表 1 与图 1 。

表1 恢复8年群落中羊草与大针茅在各个重复取样中种群个体数量 Table 1 Number of individuals in Leymus chinensis and Stipa grandis populations in each replicated plot on site of community with eight years of restoration

\begin{tabular}{ccc}
\hline $\begin{array}{c}\text { 种群 } \\
\text { Population }\end{array}$ & $\begin{array}{c}\text { 重复 } \\
\text { Replicate }\end{array}$ & $\begin{array}{c}\text { 个体数量 } \\
\text { Number of individuals }\end{array}$ \\
\hline 羊草 L. chinensis & 1 & 2601 \\
& 2 & 1869 \\
大针茅 S. grandis & 3 & 1970 \\
& 1 & 528 \\
& 2 & 512 \\
& 3 & 427 \\
\hline
\end{tabular}

数据计算使用Programita (Wiegand \& Moloney, 2004)软件完成。

\section{2 单一取样与整合重复取样条件下的点格局分析}

在研究实例中, 对于羊草种群, 在重复取样 1 中, 种群格局随着尺度的增加出现聚集分布、随机 分布和均匀分布3种类型(图2A1); 在重复取样 2 和 3 中, 种群格局在整个分析尺度上呈现聚集分布(图 $2 \mathrm{~A} 2$ 和 $2 \mathrm{~A} 3)$, 而整合 3 个重复取样后的格局在整个 分析尺度上表现为聚集分布(图2B)。对于大针茅种 群，在重复取样 1 和重复取样 3 中，种群格局随着尺 度的增加出现聚集分布和随机分布 2 种类型(图 $3 \mathrm{~A} 1$ 和 $3 \mathrm{~A} 3$ ); 在重复取样 2 中, 种群格局在整个分析尺 度上基本上呈现聚集分布(图3A2); 而整合3个重复 取样后的格局呈现聚集分布与随机分布两种类型 (图3B)。对于羊草与大针茅的种间关联，在重复取 样 1 和 2 中，两个物种在整个尺度上基本表现出种间 无关(图4A1和4A2)，尽管在某些尺度上略有偏离， 如负关联(重复取样 1 )和正关联(重复取样 2 ); 在重复 取样3中，两个物种在整个分析尺度上基本呈现正 关联(图4A3)，虽然在某些尺度上有种间无关迹象; 而整合 3 个重复取样后则在整个分析尺度上基本表 现为种间无关(图4B), 但在某些尺度上仍稍有偏离。

\section{3 讨论}

种群格局与过程一直以来都是生态学研究的 热点问题。在探讨种群格局与过程时，点格局是最 常用的方法，因其能测定不同尺度下的种群格局 (张金屯, 1998; Wiegand \& Moloney, 2004)。然而，在 众多应用点格局分析种群格局的研究实例中, 重复 取样条件下的案例非常罕见, 也就是说, 在以往的 点格局研究实例中, 研究者在取样设计时通常是在 一定尺度下选取一个群落片段，而不是同时选取几 个群落片段, 比如选取 $5 \mathrm{~m} \times 5 \mathrm{~m}$ 的群落片段, 只选
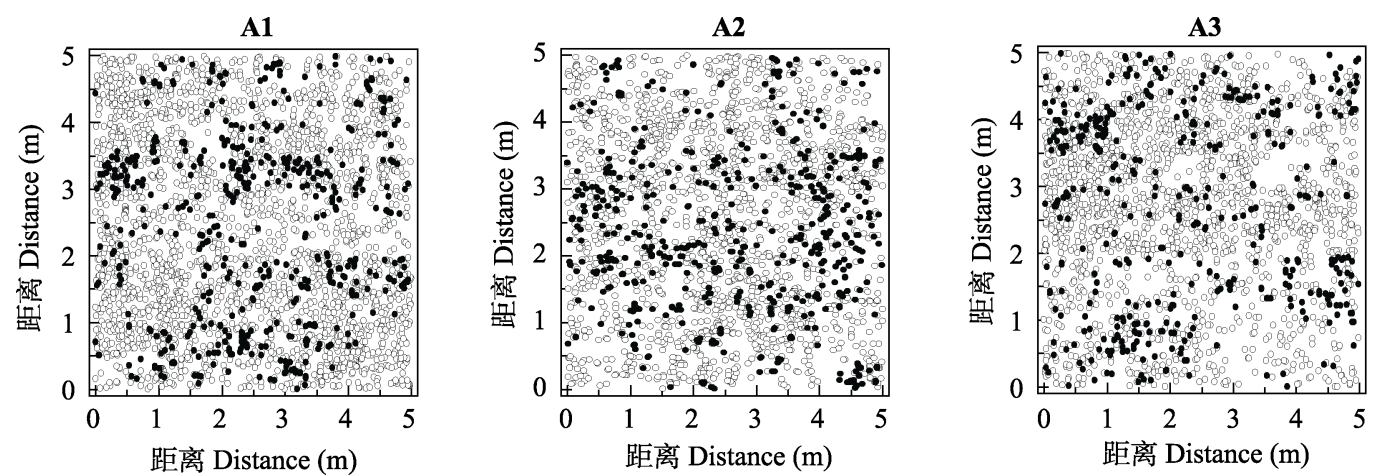

图1 恢复演替8年群落中羊草与大针茅种群的位点图(A1、A2和A3分别指重复取样1、2和3)。0, 羊草; ・ , 大针茅。

Fig. 1 Mapped points pattern of Leymus chinensis and Stipa grandis populations in communities with eight years of restoration (A1, $\mathbf{A} 2$ and A3 refer to replicates 1, 2 and 3, respectively). ○, L. chinensis; •, S. grandis.

www.plant-ecology.com 

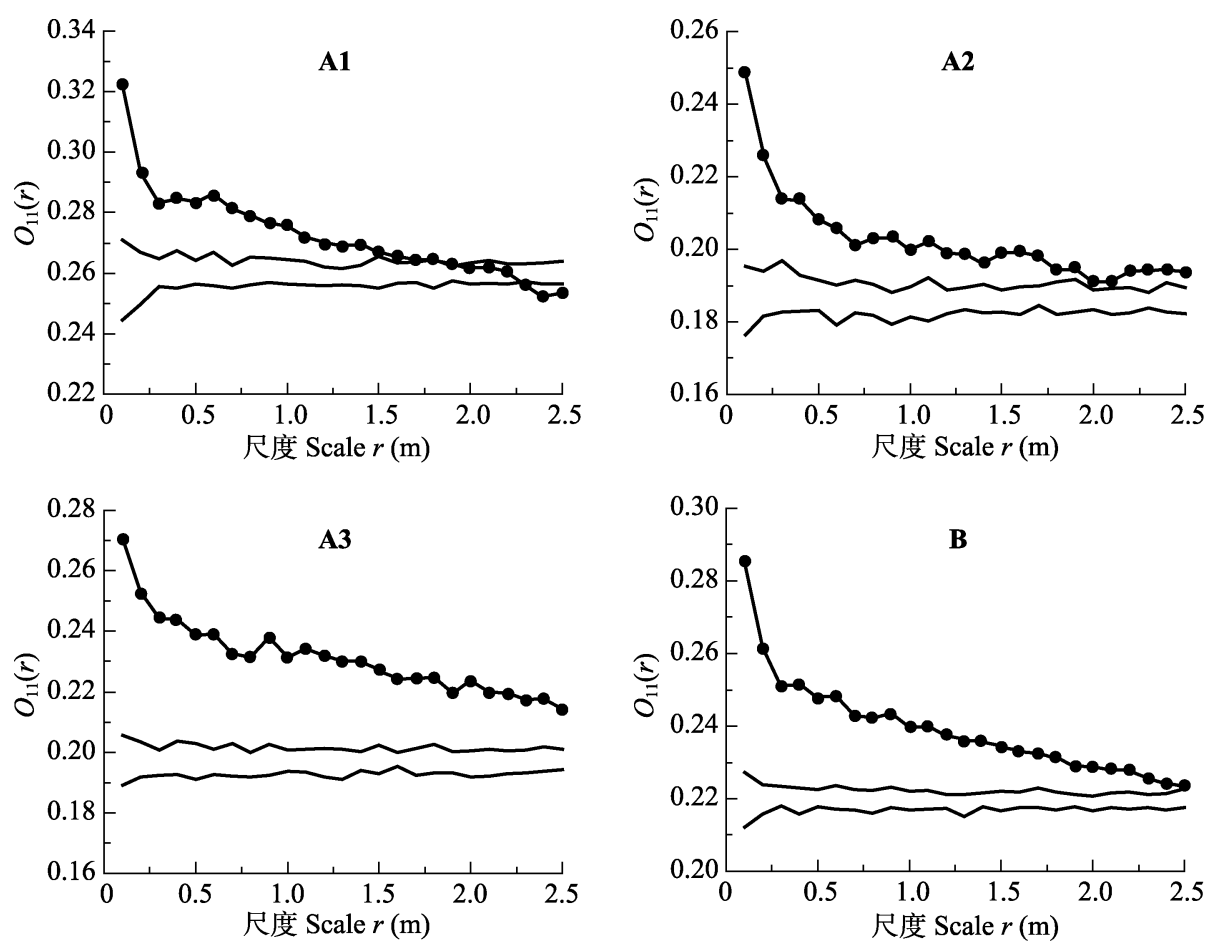

图2 恢复演替8年群落中羊草种群空间点格局分析。---, 实测数据; 一, 置信区间 $(95 \%)$ 通过完全空间随机模型获得。A, 在 每个重复中羊草种群点格局分析(A1、A2和A3分别指重复1,2和3)。B, 整合3个重复取样的羊草种群点格局分析。

Fig. 2 Point pattern analysis of Leymus chinensis population in communities with eight years of restoration. - $\bullet-$, observational data; -, the $95 \%$ confidence limits resulting from complete spatial random models. A, Point pattern analysis of $L$. chinensis population in each replicate (A1, A2 and A3 refer to replicates 1, 2 and 3, respectively). B, Point pattern analysis of L. chinensis population integrating data of three replicates.
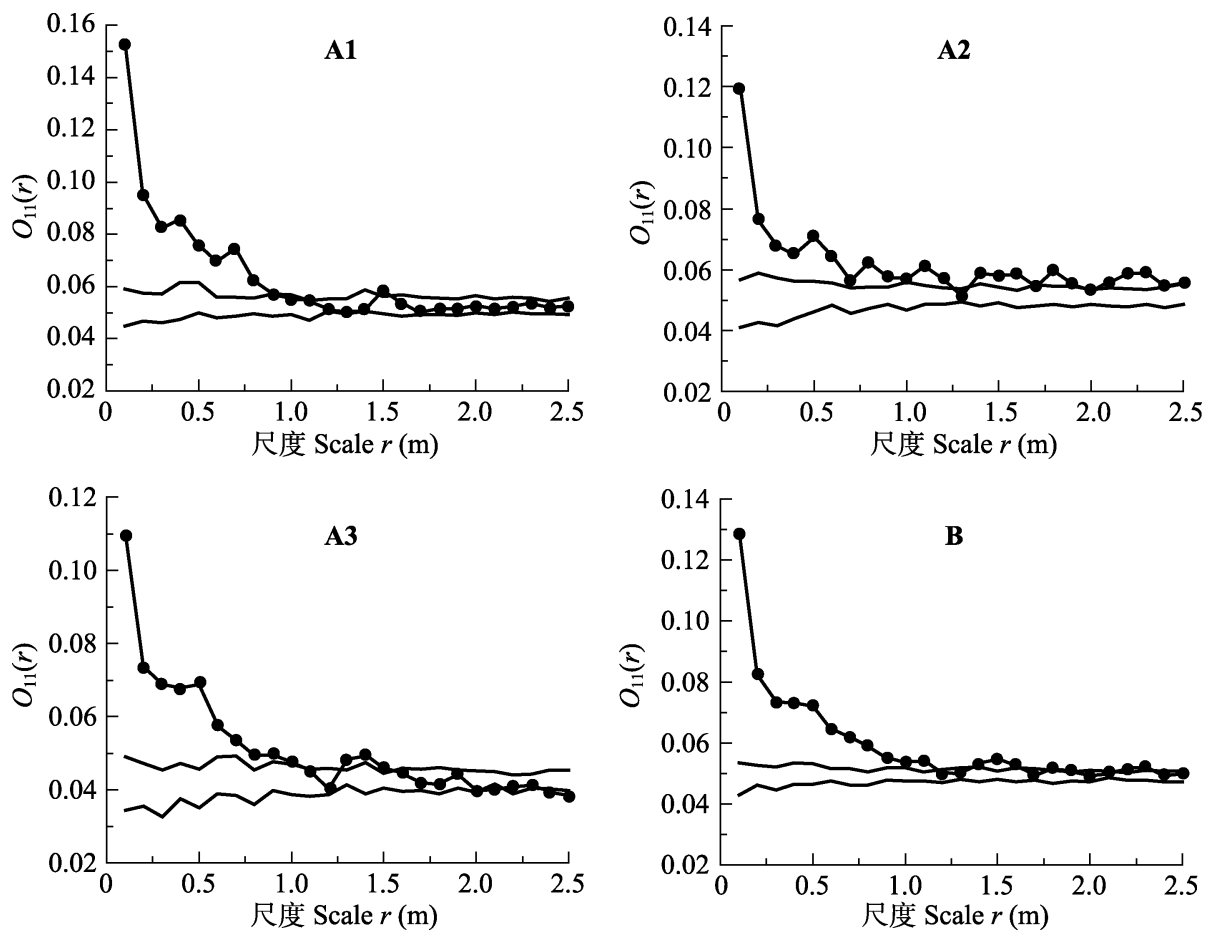

图3 恢复演替8年群落中大针茅种群空间点格局分析。-- - , 实测数据; 一, 置信区间 $(95 \%)$ 通过完全空间随机模型获得。A, 在每个重复中大针茅种群点格局分析(A1、A2和A3分别指重复1, 2和3)。 B, 整合3个重复取样的大针茅种群点格局分析。

Fig. 3 Point pattern analysis of Stipa grandis population in communities with eight years of restoration. - $\bullet-$, observational data; - , the $95 \%$ confidence limits resulting from complete randomness spatial models. A, Point pattern analysis of S. grandis population in each replicate (A1, A2 and A3 refer to replicates 1, 2 and 3, respectively). B, Point pattern analysis of $S$. grandis population integrating data of three replicates. 

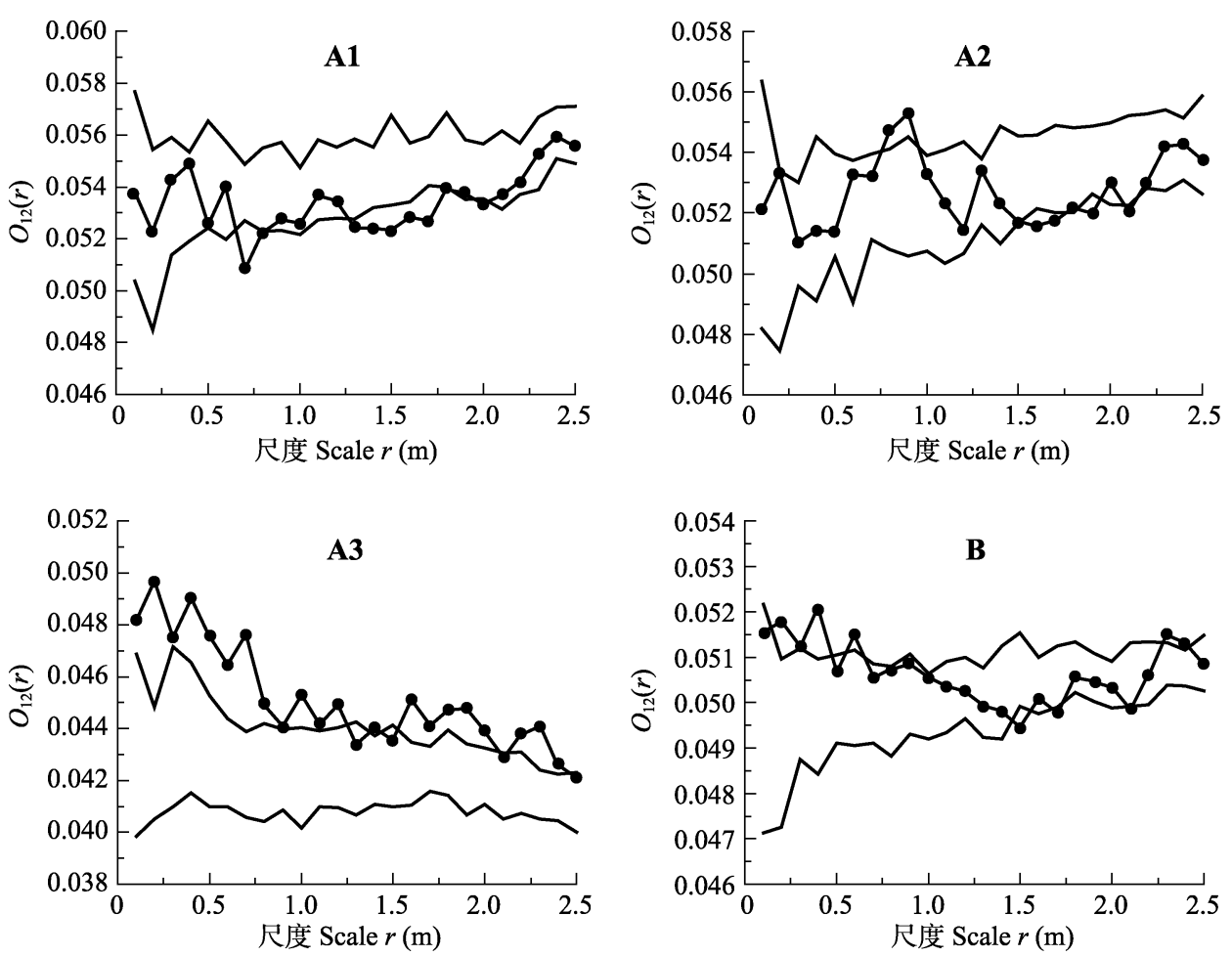

图4 恢复演替8年群落中羊草与大针茅种群种间关联分析。-- - , 实测数据; 一, 置信区间 $(95 \%)$ 通过大针茅种群固定羊草种 群随机获得。A, 在每个重复中羊草与大针茅种群种间关联分析(A1、A2和A3分别指重复1,2和 3$)$ 。 B, 整合3 个重复取样的羊 草与大针茅种群种间关联分析。

Fig. 4 Spatial association between Leymus chinensis and Stipa grandis populations in communities with eight years of restoration. $-\bullet-$, observational data; - , the $95 \%$ confidence limits resulting from fixed $S$. grandis population and random $L$. chinensis population. A, Spatial association between L. chinensis and S. grandis populations in each replicate (A1, A2 and A3 refer to replicates 1, 2 and 3, respectively). B, Spatial association between L. chinensis and S. grandis populations integrating data of three replicates.

一个而不是同时选几个, 这也是生态学研究过程中 很有意思的现象, 因为生态学家在探讨生态学问题 过程中, 重复取样是实验设计所要遵循的基本原则 之一。实际上, 对于点格局分析而言, 重复取样同样 重要, 因此, 本文提出这样的问题, 介绍重复取样 条件下点格局分析的统计学方法, 并通过实例计算 了单一取样和重复取样条件下的点格局。多个重复 条件下的点格局分析的统计学方法是加权平均 (Diggle, 2003), 对于重复条件下的点格局而言, 这 是非常有效的方法, 因为各个重复对平均状态下的 点格局的作用是不同的。每个重复的权重值是通过 每个重复中种群的个体数除以所有重复中种群个体 总数获得的。

在研究实例中, 试验在内蒙古典型草原中国科 学院草原生态系统定位研究站设置的围栏样地内进 行。在试验设计时, 为了尽量消除生境异质性对种 群格局的影响, 人为选择地表平坦、群落外貌均匀 且具有代表性的 3 个 $5 \mathrm{~m} \times 5 \mathrm{~m}$ 的群落片段。通过种 群分布的位点图(图1)可以看出, 3 个重复中, 物种空
间分布状态相似，并未表现出直观的异质性特点， 说明主观选择样地已基本排除了生境异质性的影 响。在应用点格局对每一个重复的种群格局进行分 析时，发现 3 个重复之间的格局特征存在一定差异: 羊草种群重复 1 与其他重复有差异(图2A), 大针茅 种群重复 2 与其他重复存在差异(图3A); 对种间关 联而言, 重复 3 与其他重复之间存在差异(图4A)。这 样的研究结果说明, 如果在单一取样条件下，应用 点格局探究种群格局时会出现偏差, 比如, 在研究 种群格局时, 羊草种群选择了重复1, 大针茅选择了 重复 2 , 种间关联选择了重复 3 , 据此得出的结论存 在争议，值得推敲。在研究实例中，不同重复取样间 的偏差是在主观取样尽量排除生境异质性条件后依 然存在的, 如果是随机取样，由于生境异质性的影 响, 可能偏差更大。因此, 单一取样过程中出现的试 验结果的偏差, 可能只有通过重复取样来解决, 如 整合3个重复取样的羊草种群(图2B)、大针茅种群 (图3B)及羊草与大针茅种群的种间关联(图4B)。

可见，应用点格局分析种群格局时，不同重复 
取样之间种群格局特征存在一定差异, 所以通过单 一取样得到的研究结果属于特例, 从而影响研究结 论。而整合多个重复条件下的点格局分析结果得到 的是多个重复的平均状态, 这样的状态更能体现种 群格局的整体特征而更具代表性。因为重复取样大 大增加了研究结果的稳定性, 这缘于整合多个重复 取样从某个角度而言是加大了样本量, 如此就过滤 掉了许多因样本量过小而带来的噪音, 所以, 重复 取样在样本量小时显得尤为重要, 特别适合基于多 个空间上不连续的小尺度样方的生态学研究, 比如, 在研究草原群落种群空间格局时, 取样尺度选择 $1 \mathrm{~m} \times 1 \mathrm{~m}$ 或 $2 \mathrm{~m} \times 2 \mathrm{~m}$ 的群落片段, 如果不采取重复 取样, 那么由此得到的结论其可靠性会大大降低。

另外, 重复取样的选择应因具体研究对象和问 题而异, 说到底, 重复取样问题实际上是一个尺度 问题, 如果所选取的尺度足够大, 就可能不需要重 复取样了。因此, 在选择是否重复取样时, 应根据具 体的问题、研究的尺度以及所得的样本量的大小来 决定是否需要重复取样。期望生态学研究者在应用 点格局分析种群格局时, 根据实际需要合理考虑通 过重复取样来探究格局特征。

基金项目 国家重点基础研究发展计划(973计划) (2014CB138802)、中央级公益性科研院所基本科研 业务费专项基金(1610332016002)和内蒙古自然科 学基金(2011MS0517)。

致谢 感谢内蒙古工业大学校基金(ZD201403)和内 蒙古工业大学高层次引进人才科研启动基金资助。 感谢德国赫尔霍姆茨环境研究中心的 Thorsten Wiegand博士提供Programita分析软件。

\section{参考文献}

Condit R, Ashton PS, Baker P, Bunyavejchewin S, Gunatilleke S, Gunatilleke N, Hubbell SP, Foster RB, Itoh A, LaFrankie JV, Lee HS, Losos E, Manokaran N, Sukumar R, Yamakura T (2000). Spatial patterns in the distribution of tropical tree species. Science, 288, 1414-1418.

Dale MRT, MacIsaac DA (1989). New methods for the analysis of spatial pattern in vegetation. Journal of Ecology, 77, $78-91$.

Diggle PJ (2003). Statistical Analysis of Point Patterns. 2nd edn. Arnold, London.

Galiano EF (1983). Detection of multi-species patterns in plant populations. Vegetatio, 53, 129-138.

Graff P, Aguiar MR (2011). Testing the biotic stress in the stress gradient hypothesis. Processes and patterns in arid rangelands. Oikos, 120, 1023-1030.

Greig-Smith P (1952). The use of random and contiguous quadrats in the study of structure of plant communities. Annual of Botany, 16, 293-316.

Greig-Smith P (1987). Quantitative Plant Ecology. Butterworths, London.

Jácome-Flores ME, Delibes M, Wiegand T, Fedriani JM (2016). Spatial patterns of an endemic Mediterranean palm recolonizing old fields. Ecology and Evolution, 6, 8556-8568.

Kershaw KA (1963). Pattern in vegetation and its causality. Ecology, 44, 377-388.

Pielou EC (1968). An Introduction to Mathematical Ecology. Wiley, New York.

Velázquez E, Martínez I, Getzin S, Moloney KA, Wiegand T (2016). An evaluation of the state of spatial point pattern analysis in ecology. Ecography, 39, 1-14.

ver Hoef JM, Cressie NAC, Glenn-Lewin DC (1993). Spatial models for spatial statistics: Some unification. Journal of Vegetation Science, 4, 441-452.

Wang X, Wiegand T, Hao Z, Li B, Ye J, Lin F (2010). Species associations in an old-growth temperate forest in north-eastern China. Journal of Ecology, 98, 674-686.

Wang XT, Liang CZ, Wang W (2013). Scale and density: Measuring local neighborhood density at different spatial scales. Chinese Journal of Plant Ecology, 37, 104-110. (in Chinese with English abstract) [王傘金厅, 梁存柱, 王炜 (2013). 尺度与密度：测定不同尺度下的种群密度. 植 物生态学报, 37, 104-110.]

Wang XT, Liang CZ, Wang W (2014). Balance between facilitation and competition determines spatial patterns in a plant population. Chinese Science Bulletin, 59, 1405-1415.

Wang XT, Wang W, Liu JH, Liang CZ, Zhang T (2006). A new method measuring plant population spatial patterns: Photography orientation. Journal of Plant Ecology (Chinese Version), 30, 571-575. (in Chinese with English abstract) [王釒金厅, 王炜, 刘佳慧, 梁存柱, 张蹈 (2006). 植物种 群空间分布格局测定的新方法: 摄影定位法. 植物生态 学报, 30, 571-575.]

Watt AS (1947). Pattern and process in the plant community. Journal of Ecology, 35, 1-22.

Wiegand T, Gunatilleke S, Gunatilleke N (2007). Species associations in a heterogeneous Sri Lankan Dipterocarp forest. The American Naturalist, 170, 77-95.

Wiegand T, Moloney KA (2004). Rings, circles, and nullmodels for point pattern analysis in ecology. Oikos, 104, 209-229.

Zhang JT (1998). Analysis of spatial pattern for plant species. Acta Phytoecologica Sinica, 22, 344-349. (in Chinese with English abstract) [张金屯 (1998). 植物种群空间分布的 点格局分析. 植物生态学报, 22, 344-349.]

责任编委: 沈国春 责任编辑: 王 蒇 


\section{附录}

对于Ripley’s $K$ 函数, $N$ 个重复取样条件下的数学表达式如下:

$$
\lambda K(r)=\frac{\left.\sum_{i^{1}=1}^{n^{1}} \operatorname{Points}\left[C_{i^{1}}(r)\right]\right)+\cdots+\sum_{i^{j}=1}^{n^{j}} \operatorname{Points}\left[C_{i^{j}}(r)\right]+\cdots+\sum_{i^{N}=1}^{n^{N}} \operatorname{Points}\left[C_{i^{N}}(r)\right]}{\sum_{i^{1}=1}^{n^{1}} \operatorname{Area}\left[C_{i^{1}}(r)\right]+\cdots+\sum_{i^{j}=1}^{n^{j}} \operatorname{Area}\left[C_{1, i^{j}}(r)\right]+\cdots+\sum_{i^{N}=1}^{n^{N}} \operatorname{Area}\left[C_{1, i^{N}}(r)\right]}
$$

其中, $n^{j}$ 为种群在第 $j$ 个重复取样中的个体数; $C_{i^{j}}(r)$ 为种群在第 $j$ 个重复取样中以第 $i$ 点为圆心, $r$ 为半径的圆; $\operatorname{Point}\left[C_{i^{j}}(r)\right]$ 表 示圆 $\left(C_{i^{j}}(r)\right)$ 内点的数量; $\mathrm{Area}\left[C_{i^{j}}(r)\right]$ 表示圆 $\left(C_{i^{j}}(r)\right)$ 的面积; $\lambda$ 为种群密度, 由 $N$ 个重复内个体总数除以 $N$ 个重复总面积所 得。

由于通过 $K(r)$ 既可以分析单一种群的格局特征，也可以分析两个物种的种间关联。为了区分，通常用 $K_{11}(r)$ 表示单一种群， 而用 $K_{12}(r)$ 表示两个物种, 则 $K_{11}(r)$ 与 $K_{12}(r)$ 的关于 $N$ 个重复的数学表达式为:

$$
\lambda_{1} K_{11}(r)=\frac{\left.\sum_{i^{1}=1}^{n_{1}^{1}} \operatorname{Points}_{1}\left[C_{1, i^{i}}(r)\right]\right)+\cdots+\sum_{i^{j}=1}^{n_{1}^{j}} \operatorname{Points}_{1}\left[C_{1, i^{j}}(r)\right]+\cdots+\sum_{i^{N}=1}^{n_{1}^{N}} \operatorname{Points}_{1}\left[C_{1, i^{N}}(r)\right]}{\sum_{i^{1}=1}^{n_{1}^{1}} \operatorname{Area}\left[C_{1, i^{1}}(r)\right]+\cdots+\sum_{i^{j}=1}^{n_{1}^{j}} \operatorname{Area}\left[C_{1, i^{j}}(r)\right]+\cdots+\sum_{i^{N}=1}^{n_{1}^{N}} \operatorname{Area}\left[C_{1, i^{N}}(r)\right]}
$$

其中, $n_{1}^{j}$ 为种群 1 在第 $j$ 个重复取样中的个体数; $C_{1, i^{j}}(r)$ 为种群 1 在第 $j$ 个重复取样中以第 $i$ 点为圆心, $r$ 为半径的圆; $\operatorname{Point}_{1}\left[C_{1, i^{j}}(r)\right.$ ]表示种群1在圆 $\left(C_{1, i^{j}}(r)\right)$ 内点的数量; Area $\left[C_{1, i^{j}}(r)\right.$ ]表示圆 $\left(C_{1, i^{j}}(r)\right)$ 的面积; $\lambda_{1}$ 为种群1的密度, 由种群1在 $N$ 个重复内个体总数除以 $N$ 个重复总面积所得。

$$
\lambda_{2} K_{12}(r)=\frac{\left.\sum_{i^{1}=1}^{n_{1}^{1}} \operatorname{Points}_{2}\left[C_{1, i^{1}}(r)\right]\right)+\cdots+\sum_{i^{j}=1}^{n_{1}^{j}} \operatorname{Points}_{2}\left[C_{1, i^{j}}(r)\right]+\cdots+\sum_{i^{N}=1}^{n_{1}^{N}} \operatorname{Points}_{2}\left[C_{1, i^{N}}(r)\right]}{\sum_{i^{1}=1}^{n_{1}^{1}} \operatorname{Area}\left[C_{1, i^{1}}(r)\right]+\cdots+\sum_{i^{j}=1}^{n_{1}^{j}} \operatorname{Area}\left[C_{1, i^{j}}(r)\right]+\cdots+\sum_{i^{N}=1}^{n_{1}^{N}} \operatorname{Area}\left[C_{1, i^{N}}(r)\right]}
$$

其中, $n_{1}^{j}$ 为种群 1 在第 $j$ 个重复取样中的个体数; $C_{1, i^{j}}(r)$ 为种群 1 在第 $j$ 个重复取样中以第 $i$ 点为圆心, $r$ 为半径的圆; $\operatorname{Point}_{2}\left[C_{1, i^{j}}(r)\right]$ 表示种群2在圆 $\left(C_{1, i^{j}}(r)\right)$ 内点的数量; $\operatorname{Area}\left[C_{1, i^{j}}(r)\right.$ ]表示圆 $\left(C_{1, i^{j}}(r)\right)$ 的面积; $\lambda_{2}$ 为种群 2 的密度, 由 $N$ 个重复 内种群 2 的个体总数除以 $N$ 个重复总面积所得。

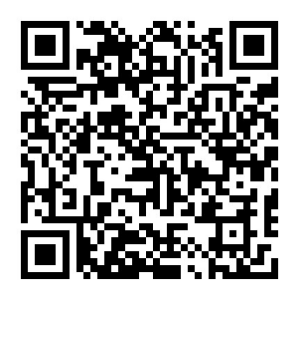

